\title{
ICE-ALE modelling for the combustion of homogeneous solid propellant
}

\author{
A. Peratta \& C. Peratta \\ Wessex Institute of Technology, Southampton, UK
}

\begin{abstract}
The paper presents a hybrid Finite Volume Method (FVM) for the laminar combustion of a double-base (homogeneous) solid propellant burning inside a two-dimensional narrowed pipe. The approach for the hydrodynamics in the gas phase is based on an Implicit-Continuous Eulerian method, implemented with the Arbitrary Lagrangian-Eulerian FVM, while the condensed phase is modelled with an Implicit Eulerian FVM approach. The hypothesis of pre-mixed laminar flame, and homogeneous solid phase are assumed. The formulation is based on the conservation equations of mass, species, momentum and energy.

The processes of degradation and pyrolysis are both taken into account in the solid phase by means of two finite chemical reactions. The complete chemical model is implemented with 7 unidirectional global finite rate chemical reactions and 12 reactive species. The outcomes of the model are pressure, temperature, density, heat release, species concentration in gas and solid phase, gas speed and bulk burning rate. The numerical model is time dependent, and it is able to describe the main production of the flame structure, including the induction (dark) zone. The numerical results obtained for the burning rate are in good agreement with the empirically based Saint-Roberts law which relates the burning rate withe the bulk pressure by means of an exponential law. The method has also been proved suitable for solving time dependent two dimensional chemically reactive flows problems presenting sub-sonic and super-sonic regimes in the same integration domain.

Keywords: combustion, solid homogeneous propellant, double-base, ale, finite volumes.
\end{abstract}

\section{Introduction}

Solid energetic materials are mostly used for rocket propulsion systems in either space or defence industry. Other applications include pyrotechnic actuators, boosters, manoeuvring and safety devices, as well as air-bags for automobile safety 
[1] and gas generators. Among the large family of solid energetic materials are the "smokeless" homogeneous propellants, developed several decades ago, which are still in use in many of the above mentioned applications. The homogeneous propellant, also known as Double-Base propellant (DBP) carries both oxidising and reducing chemical agents in the same molecular structure, usually composed of a homogeneous mixture of Nitroglycerine (NG) and Nitrocellulose (NC) $\left(\mathrm{NG}=\mathrm{C}_{3} \mathrm{H}_{5} \mathrm{O}_{3}(\mathrm{NO} 2)_{3}\right.$, and $\mathrm{NC}=\mathrm{C}_{6} \mathrm{H}_{7} \mathrm{O}_{2}\left(\mathrm{NO}_{3}\right)_{3}$, respectively) with some plasticiser and other minor ingredients [2, 3]. Because of this feature, the combustion of DBPs can be regarded as preliminary mixed from the beginning of the process. Although DBP do not usually have high specific impulses ( $I_{s} \lesssim 210 s$ under normal conditions), they are still on high demand due to its smokeless combustion, and their relatively easy manufacturing, they can be made by solvent or semi-solvent processes and extruded in various shapes. Hence, there is no need to keep into account mixing processes between oxidiser and reducer. Much effort has been done in research oriented to the CFD applied to solid propellant combustion (see for example [4, 5]). The aim of this work is to develop a comprehensive numerical modelling for the unsteady chemically reactive compressible multi-species flow interacting with the solid reactive burning surface of the DBP. The paper is organised as follows, in section 2 the theoretical formulation is established. Section 3 provides a brief description of the DB under study. Section 4 describes the interface boundary conditions, Section 5 presents the numerical scheme. Section 6 shows the numerical results obtained in a onedimensional implementation and finally Section 7 elaborates the corresponding conclusions.

\section{Mathematical formulation}

The governing equations [6] are basically the following average conservation of mass, species concentration, momentum and energy equations for a mixture of $N_{e}$ species and $N_{r}$ global chemical reactions:

Mass

$$
\frac{\partial \rho}{\partial t}+\nabla \cdot(\rho \mathrm{u})=0
$$

Species

$$
\frac{\partial\left(\rho Y_{k}\right)}{\partial t}+\nabla \cdot\left(\rho \mathrm{u} Y_{k}+\mathrm{q}_{k}^{D}\right)=\omega_{k}
$$

Momentum

$$
\frac{\partial(\rho \mathrm{u})}{\partial t}+\nabla\left(\mathrm{u}^{t} \rho \mathrm{u}+p+\bar{\sigma}\right)=0
$$

$$
\text { Energy } \quad \frac{\partial(\rho \varepsilon)}{\partial t}+\nabla \cdot\left(\rho \mathrm{u} \varepsilon+\mathrm{q}^{e}\right)+p \nabla \cdot \mathrm{u}=-\sum_{k=1}^{N_{e}} \omega_{k} \Delta h_{k}^{T_{r}},
$$

where subscript $k$ denotes species, $\omega_{k}$ is the reaction constant, $Y_{k}$ the mass fraction, $p$ pressure, $\mathrm{u}, \rho$ and $\varepsilon$ are the average velocity, density and internal energy, respectively, defined as: $\rho \mathrm{u}:=\sum_{k=1}^{N_{e}} \rho_{k} \mathrm{u}_{k}$ and $\varepsilon:=\sum_{k=1}^{N_{e}} Y_{k}\left(\varepsilon_{k}-\varepsilon_{k}^{T_{r}}\right)$, where $\varepsilon_{k}=\varepsilon_{k}^{T_{r}}+\int_{T_{r}}^{T} c_{v, k}\left(T^{\prime}\right) d T^{\prime}$, being $T$ and $T_{r}$ the actual and reference temperatures, respectively. 


\section{Chemical model, thermodynamic and transport properties}

The specific rate of $j$-th global reaction $\kappa_{j, \beta}$ is calculated by means of Arrhenius expressions of the form: $\kappa_{j}=B_{j} \exp \left[-E_{0} / R_{0} T\right]$, where $E_{0}$ is the activation energy, $R_{0}$ is the universal gas constant, and $B_{j} \approx$ cte $T^{\alpha}$ is the frequency factor with $0<\alpha<0.5$ and index $j$. Thus, $j$-th rate reaction can be calculated by means of:

$$
\omega_{j}^{R}=\kappa_{j, f} \prod_{k=1}^{N_{e}} C_{k}^{\nu_{k j}^{*}},
$$

where $\nu_{j k}^{*}$ is the stoichiometric coefficient of the reactive species $k$ in reaction $j$, and $C_{k}$ is the mole concentration of the species.

The following equation of state (EOS) is considered in the gas phase

$$
p=\rho R_{0} T \sum_{k=1}^{N_{e}} Y_{k} / M_{k}
$$

where $c_{s}^{2}=p / \rho\left(1+R_{\text {mix }} / c_{v \text { mix }}\right) ; M_{k}$ is the molecular mass, $R_{\text {mix }}:=$ $R_{0} \sum_{k=1}^{N_{e}} Y_{k} / M_{k}$ the mixture constant, $c_{v \text { mix }}=\sum_{k=1}^{N_{e}} Y_{k} c_{p k}-R_{\text {mix }}$ the mixture specific heat, and $c_{s}$ the speed of sound. The condensed phase $(\mathrm{CP})$ is considered incompressible, i.e. infinite sound speed. The specific heat at constant pressure of each component is evaluated using: $c_{p k}(T)=A_{k}+B_{k} T+C_{k} T^{2}+D_{k} T^{3}+E_{k} / T^{2}$, where $A, B, C, D$ and $E$ are constants obtained from [7, 8]. Then, the specific internal energy of the mixture become: $c_{v \text {, mix }}=\sum_{k=1}^{N_{e}} Y_{k} c_{p k}-R_{\text {mix }}$.

Transport coefficients of species involved in energetic materials are not always available in published tables, therefore, they have to be approximated from the classical statistical theory of gases. The diffusive effects considered in eqs. (1) to (4) are viscosity, Fick's diffusion and Fourier law for heat conduction. In this way $\mathrm{q}_{k}^{D}=-\rho D_{k, \text { mix }} \nabla Y_{k}$ in (2) is the Fick's mass diffusive flux, $\sigma$ is the viscous tensor in (3), and $\mathrm{q}^{e}=-\lambda_{\operatorname{mix}} \nabla T-\rho \sum_{k=1}^{N_{e}} h_{k} Y_{k} u_{k}-\nabla(\bar{\sigma} \mathrm{u})$ is the diffusive flux of internal energy. The binary diffusion coefficient between species $\mathrm{A}$ and $\mathrm{B}$ is calculated by mean of the Chapman-Enskog $(\mathrm{C}-\mathrm{E})$ theory and the transport Boltzmann equation [9].

For the multi-component mixture, the effective diffusion coefficient ( $\left.D_{k, \text { mix }}\right)$ of $k$ with respect to the mixture is related to binary one $D_{A B}$ by means of Wilke's mixing rule $[9,10]$ :

$$
D_{k, \text { mix }}=\left(1-X_{k}\right) /\left(\sum_{j \neq k}^{N_{e}} \frac{X_{j}}{D_{k j}}\right) .
$$

Viscosity for a single component is calculated using C-E theory and L-J potential according to: $\eta=5 / 16 \sqrt{\pi m k_{B} T} /\left(\pi \sigma^{2} \Omega_{D}\right)$. C-E theory can also be applied to obtain the thermal conductivity $\lambda$ of pure mono-atomic species with no internal degrees of freedom [9, 11], yielding $\lambda=25 / 32 \sqrt{\pi m k_{B} T} c_{v} /\left(\pi \sigma^{2} \Omega_{D}\right)$. Then, Eucken's correction factor given by $\lambda M \eta^{-1}=1.32 c_{v}+1.77 R_{0}$, is used to consider the internal degrees of freedom in polyatomic molecules, and finally, for the multi-component mixture, Wilke's rule is used in the same way as for the viscosity coefficient $(\eta)$ above. Reference values for the calculation of cross 
sections, dipole moments, and collision integrals can be obtained from refs. $[9,11,12]$.

\section{The DB propellant}

In this work the $\mathrm{EC} 1$ formulation $(52 \% \mathrm{NG}+43 \% \mathrm{NC}+5 \%$ of minor additives $)$ contemplated by A. Kubota [2], with $1100 \mathrm{cal} / \mathrm{g}$ of heat of explosion has been adopted. Because of its relatively large stability under room conditions, an external source of heat (igniter) must be provided to initiate the DBP burning. During the ignition process, heat is transported by conduction inside the solid and the degradation reaction of $\mathrm{NG}$ and $\mathrm{NC}$ begins to accelerate generating reactive species like $\mathrm{NO}_{2}$ and aldehydes.

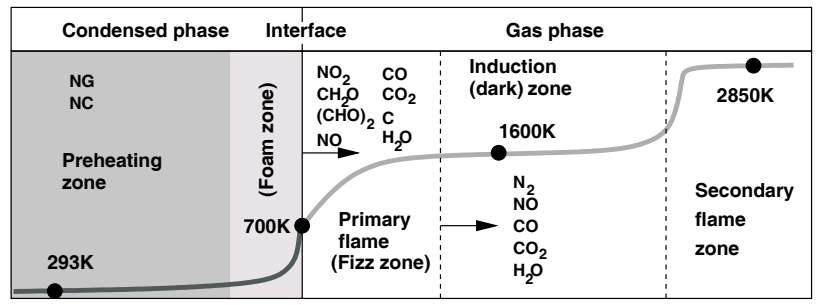

Figure 1: Temperature, species and regions in condensed and gas phase near the burning surface of the DBP.

Then, further recombination takes place into the solid phase, yielding extra heat to reinforce the ignition. We call condensed phase (CP)to the region between gas and solid phase where solid propellant coexists with degradation and recombination products.(The $\mathrm{CP}$ consists of a pre-heated zone and a superficial degradation layer where molecular degradation of the propellant and further recombination of the decomposed products takes place.) As degradation evolves, the solid concentration drops down from one to zero. The closer to the burning surface the higher degradation rates. When solid concentration reaches zero at the interface, convection process drags the unburnt condensed phase from the cold zone of the DBP thus replacing the decomposed material. Then, a convective chemically reacting flow emerges from the burning surface toward the gas phase with a velocity ruled by eq.(1). The emerging molecules react with each other in the gas phase, developing an important amount of heat in the flame zone. The distribution of heat release along the flame zone is ruled by the coupling between the gas-phase reactions, hydrodynamics, and transport phenomena, thus yielding a particular flame structure as qualitatively shown in Fig. 1. Meanwhile, the heat released in the primary zone provides some feedback energy to maintain the degradation reaction in the $\mathrm{CP}$. The combustion can be either self-sustained steady, unsteady, oscillatory, or vanishing, depending on this energetic loop. The modelling involves $N_{r}=7$ chemical reactions, $[10,12]$ and the following chemical species: $\mathrm{DB}, \mathrm{NO}_{2}, \mathrm{CH}_{2} \mathrm{O},(\mathrm{CHO})_{2}, \mathrm{CO}, \mathrm{NO}, \mathrm{CO}_{2}$, 
$\mathrm{H}_{2} \mathrm{O}, \mathrm{H}_{2}, \mathrm{C}_{2} \mathrm{H}_{4}, \mathrm{~N}_{2}$ and $\mathrm{C}$. The corresponding rate reactions, specific enthalpy, and Arrhenius coefficients were obtained from [12-14].

\section{Interface boundary conditions}

Gas and CP equations couple to each other throughout the burning surface (interface) by means of the following mass and species conservation equations:

$$
[\rho u]_{\mathrm{gas}}=\rho_{p} r_{b}, \quad \text { and } \quad\left[\rho \mathrm{u}_{k} \cdot \hat{n} Y_{k}\right]_{\mathrm{gas}}=\rho_{p} r_{b} Y_{k},
$$

respectively, and energy according to:

$$
\left[-\lambda_{\operatorname{mix}} \frac{\partial T}{\partial \hat{n}}+\rho \sum_{k=1}^{N_{e}} Y_{k} h_{k} \mathrm{u}_{k} \cdot \hat{n}\right]_{\mathrm{gas}}=-\lambda_{s} \frac{\partial T}{\partial \hat{n}}+\rho r_{b}\left[c_{p}\left(T_{s}-T_{r}\right)+\sum_{k=1}^{N_{e}} Y_{k} \Delta h_{k}\right]_{s}
$$

where subscript $s$ refers to the $\mathrm{CP}, r_{b}$ is the local propellant burning rate, $T_{s}$ is the interface temperature, $h$ is enthalpy and $\Delta h$ the enthalpy at reference temperature. Because reactions in the $\mathrm{CP}$ have relatively high activation energy and occur in a very thin region (few microns in foamy zone), a matched asymptotic expansion technique as described in refs. $[15,13,14]$ can be used in order to estimate the burning rate formula. The result for a steady self-sustained combustion is:

$$
r_{b}^{2} \approx \beta^{\prime} \frac{T_{s}^{2}}{T_{s}-T_{0}-Q_{s} /\left(2 c_{s}\right)} \exp \left[\frac{-E_{c}}{R_{0} T_{s}}\right]
$$

where $\beta^{\prime}:=\lambda_{s} B c R_{0} /\left(c_{S} \rho_{s} E_{c}\right)$ is a constant, $T_{0} \approx 293 K$ is the initial temperature equal to the temperature at $x \rightarrow \infty$ in the cold zone of the propellant. The following values were assumed: $\rho_{s}=1.6 \mathrm{~g} / \mathrm{cm}^{3}, c_{s}=0.4 \mathrm{cal} /(\mathrm{gK}), \lambda_{s}=$ $5.1 \times 10^{-2} \mathrm{cal} /(\mathrm{smK}), B_{c}=10^{17} \mathrm{~s}^{-1}$, and $E_{c}=40 \mathrm{kcal} / \mathrm{mol}$. Thus $r_{b} \approx$ $10^{-2} \mathrm{~m} / \mathrm{s}$, and consequently: $\delta x_{c} \approx 37 \mu \mathrm{m}, \tau_{c} \approx 4 m s, \delta x_{d} \approx 2 \mu m, \tau_{d} \approx 0.2 \mathrm{~ms}$, where $\delta x$ indicates characteristic length, $\tau$ residence time, and subscripts $c$ and $d$ pre-heated and foamy zone in the $\mathrm{CP}$, respectively. Note that the typical Mach number in the flame zone is $M \approx 10^{-3}$, this involves an incompressible flow regime which might potentially limit the numerical scheme.

\section{Numerical scheme}

The gas phase is solved with the Implicit Continuous Eulerian (ICE) method [16] and an Arbitrary Lagrangian-Eulerian (ALE) technique. The region involving the solid (condensed)-gas phase interface has been modelled with a one-dimensional approach oriented perpendicular to the burning boundary. The scheme for both gas and condensed phases is based on the FVM in which each physical process is integrated in an independent way during one calculation time step. The FVM is implemented with a staggered grid approach where diffusive fluxes (q) are assigned to cell faces, flow velocities $(u)$ to cell vertices and the other field variables such as $\varepsilon_{\text {mix }}, T, p$, cell volume $V$, and $Y_{k}$ are assigned to cell centres. The different objects participating in the discretisation are basically cell vertices, 
faces and centres. An update of the cell dynamic property $\psi$ between time level $m$ and $m+1$ is generally written as: $\psi^{m+1}=\psi^{m}+\delta \psi_{D}+\delta \psi_{H}+\delta \psi_{S}$, where $\delta \psi_{D}, \delta \psi_{H}$, and $\delta \psi_{S}$ represent the contribution to the total variation of $\psi$ due to diffusive, hydrodynamic and source terms, respectively. Time variations $\delta \psi_{D}$ of either mass or energy due to either Fick and Fourier diffusion, or species migration is calculated in a Lagrangian way with an Alternating Direction Implicit (ADI) approach [17]. During the ADI step, all other terms contributing to the variation of $\psi$ apart from the diffusive ones, are considered as frozen. The ICE approach [12, $16]$ is employed in order to update field variables due to the hydrodynamic process $\left(\delta \psi_{H}\right)$, i.e. for integrating the pressure-velocity coupling and the kinetic energy of the system. Time variations due to source terms $\left(\delta \psi_{S}\right)$ are integrated explicitly in time. For example, the increment of species concentration and total internal energy in the cell, due to the source terms appearing in (2) and (4) arising from chemical reactions become: $\delta Y_{k, i}^{m+1}=\frac{\omega_{k, i}^{m}}{\rho_{i}^{m}} \delta t, \quad$ and $\delta(m \varepsilon)_{i}^{m+1}=V_{i}^{m} \sum_{k=1}^{N_{e}} \omega_{k, i}^{m} \Delta h_{k} \delta t$, respectively, where $m_{i}$ and $V_{i}$ represent the total mass and volume of cell $i$.

During the Lagrangian step, all convective terms are zero, and the vertices move with the fluid in order to preserve constant the total mass inside a cell. Because of that, after a few time steps the grid may become excessively distorted. In addition the cells near any outflow boundary condition may result pushed away from the integration domain. In order to overcome these technical difficulties all mesh vertices are moved backwards, i.e. usually opposite to the flow motion, with an arbitrary numerical velocity $\mathrm{u}_{\mathrm{a}}$ given by the superposition:

$$
\mathrm{u}_{\mathrm{a}}=-\mathrm{u}+\tau\left(\frac{\mathrm{x}_{\mathrm{i}}^{*}-\mathrm{x}_{\mathrm{i}}}{\delta t}\right)
$$

where $\mathrm{u}$ is the fluid velocity, $\mathrm{x}_{i}^{*}$ are the $2 \mathrm{D}$ coordinates of the grid vertices at the new time level, $\mathrm{x}_{\mathrm{i}}$ represent the actual location of the vertices, and $\tau$ is a relaxation factor. When $\tau=0$ the method resembles an Eulerian one. The second term in the right hand side of eq. (7) represents an arbitrary velocity which is added in order to adapt the grid space resolution to the gradient of the developing solution. The new location of the grid points is computed in order to obtain a uniform distribution of the Lagrangian coordinates $L_{i}$ associated with $i$-th cell, i.e.:

$$
L_{i}=\int_{0}^{x_{1}^{*}} \int_{0}^{x_{2}^{*}} w\left(\mathrm{x}^{\prime}\right) d x_{1}^{\prime} d x_{2}^{\prime}=\frac{1}{N_{c}},
$$

where $N_{c}$ is the number of cells, and $w=c\left(1+\nabla \cdot\left(b_{1} T+b_{3} Y_{C O_{2}}\right) b_{2} \omega_{k} \Delta h_{k}\right)$ is a density distribution function, $b_{1}, b_{2}$ and $b_{3}$ are scaling constants, and $c$ is a normalisation factor that makes $\sum_{i=1}^{N_{c}} L_{i}=1$ the integral (8) over the whole integration domain. As a consequence, convective terms are artificially restored, and the mesh tends to increase its resolution in regions with steep gradients of $\mathrm{CO}_{2}$ concentration and temperature and local peaks of heat release. A detailed explanation of the scheme and the corresponding pseudo-code is presented in $[10,12]$. 


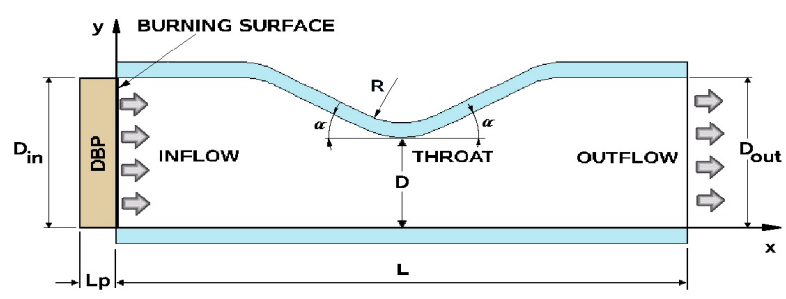

Figure 2: Conceptual model.

\section{Results}

The ICE-ALE FV method has been applied to the combustion of a DBP providing inlet flow conditions to the two-dimensional domain shown in Fig. 2. The geometrical parameters are length $L=16 \mathrm{~cm}$, inflow and outflow aperture $D_{\text {in }}=D_{\text {out }}=5 \mathrm{~cm}$, convergent and divergent angle $\alpha=30^{\circ}$. The throat width and curvature radius are $D=3 \mathrm{~cm}$ and $R=2 \mathrm{~cm}$, respectively.

The burning surface is located at $x=0$ where the FV version of matching conditions (5), (6) are prescribed. The cold end of the DBP has $T=293 \mathrm{~K}$, $Y_{\mathrm{DB}}=1$, and $Y_{k}=0$ for $k \neq D B$. The top and bottom surfaces are considered as adiabatic walls. The corresponding boundary conditions are implemented by mean of phantom cells and vertices. Smooth outflow boundary conditions like $\partial\left(u_{x}, \varepsilon, Y_{k}\right) / \delta x=0$ are prescribed at the gas exit. The initial conditions are $Y_{\mathrm{DB}}=1, Y_{\mathrm{N} 2}=0, T=293 \mathrm{~K}$ at the DB propellant, and $Y_{\mathrm{DB}}=0, Y_{\mathrm{N} 2}=1$, $T=650 K$ at the gas phase. An external source of heat has been added in order to maintain a temperature of $650 \mathrm{~K}$ during $1 \mathrm{~ms}$ so that pyrolisys and decomposition endotermic reactions in the solid can start relatively quickly. This external source can be regarded as a simplified representation of an hypothetical ignition system. This transient condition is removed after the combustion becomes self sustained. It has been observed from the numerical experiments that all transient effects occurring near the burning front due to ignition and flame structure development vanish after approximately $t=0.2 s$ when the process becomes stable. Fig. 3 shows the concentration profile of all species in gas phase and $\mathrm{CP}$ at $t=0.2 \mathrm{~s}$.

The main properties of steady state flame structure is relatively insensible to the geometry and physical conditions of the bulk of the domain, apart from the bulk pressure which affects the characteristic length scale of the flame. The results obtained for temperature and species concentration are in excellent agreement with the ones previously obtained in [14] and others qualitative descriptions of the flame structure [15].

Figure 4 shows the temperature distribution, and its development towards the steady state regime. At time $t=0.09543 \mathrm{~s}$ a pseudo-steady state is achieved for the temperature distribution.

The characteristic velocity near the burning zone is of the order of a few $\mathrm{m} / \mathrm{s}$, and the temperature is typically $700^{\circ} \mathrm{C}$. Therefore the Mach number is of the order $10^{-3}$, thus representing a quite incompressible behaviour. 

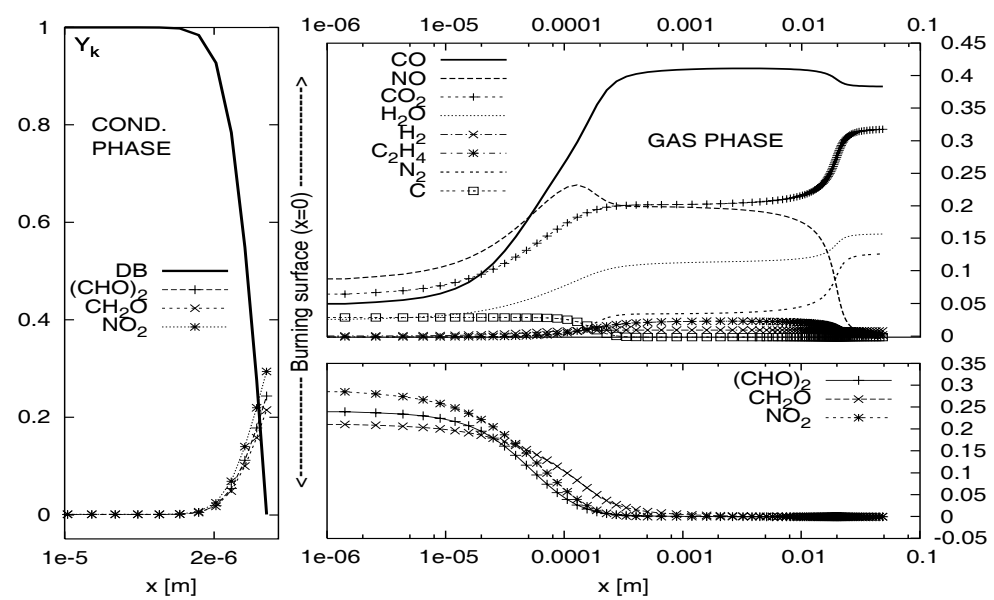

Figure 3: Species concentration profile near the burning surface in gas and condensed phases at $t=0.2 \mathrm{~s}$.
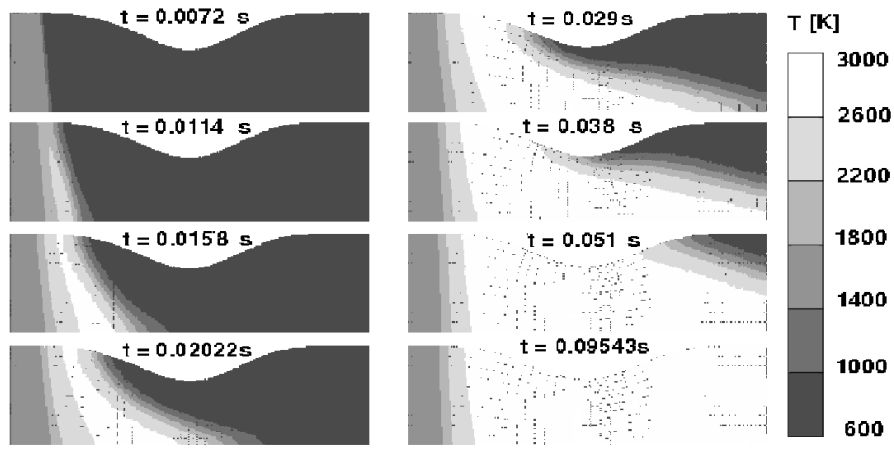

Figure 4: Temperature at different time levels after the ignition.

In order to test the ability of the ICE method to deal with chemically reacting subsonic, transonic and supersonic regime at the same time, the DBP was replaced by a constant inflow boundary condition supplying hot reactive gases at $450 \mathrm{~m} / \mathrm{s}$. Note that this is an extraordinary high value in comparison with the typical velocity found in the gas phase near the burning surface. However, it is useful as a benchmark test for the hydrodynamic part of the problem. The chemical composition of the flow at the inlet has been imposed in order to match the corresponding flame composition at steady state in the interface, found in onedimensional simulations (see Table 1). The temperature at the inlet section was fixed to $700 \mathrm{~K}$.

Figure 5 shows the Mach number $\left(M=|u| / c_{s}\right)$ distribution in the steady state. As expected, the transonic region is located in the narrowed section of the domain, the convergent region is subsonic and the convergent region is supersonic. 
Table 1: Mass concentration of the inlet flow.

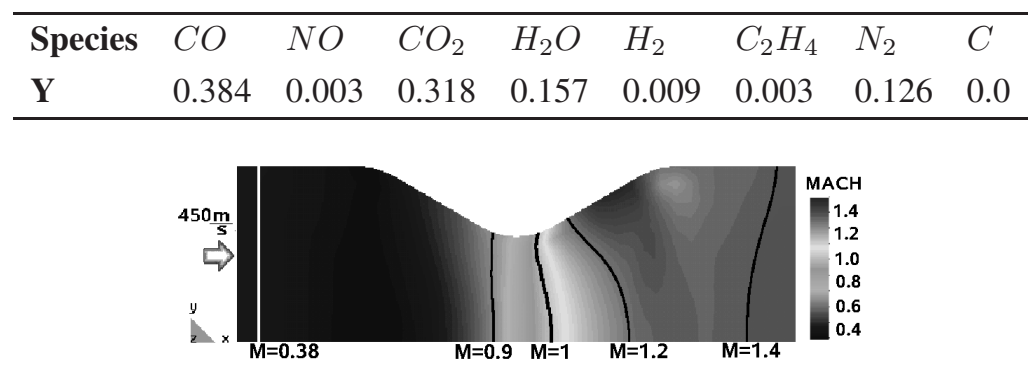

Figure 5: Mach number in a compressible regime.

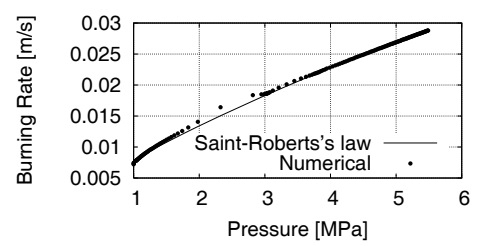

Figure 6: Burning rate vs. pressure. Comparison between numerical finding and the Saint-Robert's law.

Figure 6 shows the burning rate plotted against the bulk pressure. The dots represent the values explored during the numerical calculation, while the continuous line represents the Saint-Robert's law (Vieille's law) given by $r_{b}=$ $A\left(p / p_{0}\right)^{n}$. The exponent $n$ which best fits the numerical relation in a least squares sense is $n=0.76$. This value is in good agreement with the expected indices for DBPs.

\section{Conclusions}

A hybrid approach based on the FVM was developed and tested in order to study time dependent combustion problems involving homogeneous Double-Base propellants. The method is based on an Implicit Continuous Eulerian formulation with an Arbitrary Lagrangian Eulerian technique and mesh optimisation. The present approach is in principle oriented to compressible flow, however, it resulted quite satisfactory for modelling very low Mach numbers, i.e. order $O\left(10^{-3}\right)$. The method is suitable for dealing with subsonic, transonic and supersonic regimes at the same time. After the flame has achieved a steady state, the comparison between Saint-Robert's burning law and the numerical findings are very good. In addition, the scheme has proven to be stable and provided accurate description of the flame structure and flame dynamics near the burning surface of the propellant. Hence, it can be used to study a variety of 2D time dependent combustion processes including transient ignition and combustion instabilities. 


\section{References}

[1] P. P. Cord and F. Beteille. Versatility of a two chamber inflator for airbag application. In Airbag 2004, 7th Int. Symposium and exhibition on Sophisticated car Occupant Safety Systems, Karlsruhe, Germany, 2004.

[2] Aoki N. Kubota. Combustion wave structures of high - and low energy double-base propellants. AIAA J., 20(1):100-105, 1982.

[3] E. Dick, J. Bulgheroni, R. Crespo, J. Padula, E. Runstein, and L. Urdiain. La propulsion de cohetes y misiles (in spanish). Technical Report 685, CITEFA, Bs As, Argentina, 1989.

[4] M. T. Health, R. A. Fiedler, and W. A. Dick. Simulating solid propellant rockets at csar. AIAA Paper, pages 2000-3455, 2000.

[5] H. J. Gibeling, R. K. Madabhushi, F. J. De Long, and J. S. Sabnis. Advanced flow field model. Technical report, U.S.Air Force Philips Lab., 1993. CRPLTR-3005, Edwards AFB CA.

[6] J. Warnatz, U. Maas, and R.W. Dibble. Combustion. Springer Verlag, 1996.

[7] D.R. Stull and H. Prophet. Janaf tables of thermochemical properties, 2nd ed. Technical report, National Standard Ref. Data System, Washington, 1971.

[8] Nat. Inst. of Standards and Tech. The NIST Chemistry WebBook. http: / / webbook. nist.gov/,2005. U.S. Secretary of Commerce.

[9] Hirschfelder, Curtis, and Bird. Molecular Theory of gases and Liquids. John Wiley \& Sons, Inc., New York, 1954.

[10] A. Peratta, C. González, and E. Dick. Numerical analysis for double-base propellant combustion. In V. Sanchez-Galvez and C.A. Brebbia, editors, Computational Ballistics II, volume 40 of Transactions on Modelling and Simulations, pages 269-278. Wessex Institute, WIT-Press, 2005.

[11] B. E. Poling, J. M. Prausnitz, and John P. O' Connel. The Properties of gases and Liquids. McGraw Hill, 5 edition, 2000.

[12] A. Peratta. CFD Aplicado a la Combustión de Propulsantes Sólidos Homogéneos. Master's thesis, University of Buenos Aires, Faculty of Exact and Natural Sciences, October 2001. in spanish.

[13] V. Yang, Thomas Brill, and Wu-Zhen Ren, editors. Solid Propellant Chemistry, Combustion, and Motor interior Ballistics, volume 185. American Institute of Aeronautics and Astronautics, New York, 2000.

[14] Tae-Seong Roh, I-Shih Tseng, and Vigor Yang. Effects of acoustic oscillations on flame dynamics of homogeneous propellants in rocket motors. J. of Propulsion and Power, 11(4), July August 1995.

[15] G. Lengellé, A. Bizot, J. Duterque, and J.F. Trubert. Fundamentals of solid propellant combustion. In K.K.Kuo and M. Summerfield, editors, Progress in Astronautics and Aeronautics, AIAA, volume 90, page 361, New York, 1984.

[16] C.W. Hirt, A.A. Amsdem, and J.L.Cook. An arbitrary lagrangian-eulerian method for all flow speeds. J. of Computational Physics, 14:227-253, 1974.

[17] G.I. Marchuk. Handbook of Numerical Analysis, volume 1, chapter Splitting and alternating direction methods, pages 197-462. Elsevier, North-Holland, Amsterdam, 1990. 